LA W RENCE LIVERMORE NATIONAL LABORATORY

J. Pruet, D. P. McNabb

February 16, 2006

T-REX Design Considerations for Detection of Concealed 238U 
This document was prepared as an account of work sponsored by an agency of the United States Government. Neither the United States Government nor the University of California nor any of their employees, makes any warranty, express or implied, or assumes any legal liability or responsibility for the accuracy, completeness, or usefulness of any information, apparatus, product, or process disclosed, or represents that its use would not infringe privately owned rights. Reference herein to any specific commercial product, process, or service by trade name, trademark, manufacturer, or otherwise, does not necessarily constitute or imply its endorsement, recommendation, or favoring by the United States Government or the University of California. The views and opinions of authors expressed herein do not necessarily state or reflect those of the United States Government or the University of California, and shall not be used for advertising or product endorsement purposes.

This work was performed under the auspices of the U.S. Department of Energy by University of California, Lawrence Livermore National Laboratory under Contract W-7405-Eng-48. 


\title{
T-REX Design Considerations for Detection of Concealed ${ }^{238} \mathrm{U}$
}

\author{
J. Pruet \& D.P. McNabb \\ February 16, 2006
}

\begin{abstract}
Here we outline considerations that might inform choices for the design of a laser/linac-based light source used to detect ${ }^{238} \mathrm{U}$ via excitation of the resonance at $680.11 \mathrm{keV}$ in this isotope. We assume that the principal concern is speed of interrogation and not, e.g., how much radiological dose is imparted during a scan. It is found that if the photon detectors used in the system have an energy resolution better than or comparable to that of the interrogation beam, then to a first approximation the light source should be designed to have the highest possible specific fluence (photons per unit energy per unit time). There is also a weak dependence of scan time on the number of photons emitted per pulse of the light source. A simple formula describing the tradeoff between specific fluence and number of photons per pulse is presented.
\end{abstract}

\section{Introduction}

A T-REX system that will be used to demonstrate detection of hidden ${ }^{238} \mathrm{U}$ in laboratory experiments is now being developed. Assuming that successful completion of these experiments is the most important consideration, it seems that the prototype interrogation system should be designed so as to allow interrogation in the shortest possible time. Here we describe how the T-REX system should be designed so as to minimize interrogation time. For systems that are deployed in practical situations, such as ports or medical screening, other considerations like radiological dose might be more important. 


\section{Interesting Light Source Characteristics}

Three factors affect how quickly a sample can be scanned. The first is the on-resonance brightness $b$ of the light source. Here $b$ has units of photons per unit energy per unit time. Since estimates of the angular divergence of the source are so small (sub milliradian), the precise value of the angular spread is not really important. The second light-source property influencing measurements is $\Gamma_{b}$, the energy resolution of the beam. Here $\Gamma_{b}$ has units of energy and can be defined as the full-width at half max of the spectrum. The last factor influencing measurements relates to the repetition rate $r$ of the light source. Here $r$ is the number of light pulses emitted per unit time, so that $b \Gamma_{b} / r$ is the number of photons emitted per pulse.

\section{Signal Collection Rate}

A key quantity is the rate at which resonant photons are observed in the notch detector. For reference we note that the $680 \mathrm{keV}$ resonance in ${ }^{238} \mathrm{U}$ decays about $56 \%$ of the time via emission of a $635 \mathrm{keV}$ photon, and about $44 \%$ of the time via emission of a $680 \mathrm{keV}$ photon. Both photons can be usefully detected.

Assuming that clandestine material is not present in the interrogated sample (a suitcase), the signal collection rate is

$$
\lambda_{\mathrm{NRF}}=b \Gamma_{\mathrm{NRF}} \exp \left(-\tau_{\text {luggage }}-\tau_{680}^{\mathrm{Pb}}\right) \epsilon_{d} .
$$

Here $\tau_{\text {luggage }}$ is the optical depth of the examined suitcase to photons with $\mathrm{E} \sim 680 \mathrm{keV}, \epsilon_{d}$ is the efficiency of the notch detection system and

$$
\Gamma_{\mathrm{NRF}} \approx 1 \mathrm{eV}
$$

is the effective width of the NRF resonance in ${ }^{238} \mathrm{U}$. In eq. 1 we have also included the possibility that lead or some other attenuating material will be placed between the sample foil and the photon detector. The optical depth of this material to $680 \mathrm{keV}$ photons is represented by $\tau_{680}^{\mathrm{Pb}}$.

\section{Background collection rate}

It is convenient to distinguish between two kinds of backgrounds. The first is comprised of photons that elastically scatter from the Uranium foil in 
the notch detector. If the energy of these photons is close enough to 680 $\mathrm{keV}$, they cannot be distinguished from the $680 \mathrm{keV}$ photons emitted during decay of resonantly excited nuclei in the sample foil, though they could be distinguished from the $635 \mathrm{keV}$ photons.

The second kind of background is comprised of photons that undergo Compton scattering in the sample foil. Since these photons lose a fair chunk of their initial energy, they can (with an energy resolving detector) be distinguished from NRF photons. However, they can still lead to pulse summing in the detection system and in this way might confuse detection.

When Bremsstrahlung sources are used there is another type of background that becomes important. This arises from multiple scattering processes in the sample foil and is rather complicated. These backgrounds cannot be confused with the NRF signal when a narrowband light source is used and do not appreciably contribute to pulse pileup, so they will not be discussed here.

\subsection{Elastic Backgrounds}

Photons with energy near $680 \mathrm{keV}$ have an effective cross section for large angle elastic scattering of $\sigma_{\text {elastic }} \approx 5 \cdot 10^{-2}$ barns. Noting that the NRF cross section for exciting the $680 \mathrm{keV}$ state in uranium is $\sigma_{\mathrm{NRF}} \approx 143$ barns, we find that the relative rate at which elastically scattered background photons is collected is

$$
\frac{\lambda_{\text {elastic }}}{\lambda_{\mathrm{NRF}}}=\frac{\sigma_{\text {elastic }}}{\sigma_{\mathrm{NRF}}} \times \frac{\Gamma_{\text {expt }}}{\Gamma_{\mathrm{NRF}}} \approx 0.25 \times \frac{1 \mathrm{keV}}{\Gamma_{\text {expt }}} .
$$

Here $\Gamma_{\text {expt }}$ is an effective energy resolution for the detection setup. If the light source has a resolution smaller than the resolution of the notch system, then $\Gamma_{\text {expt }}=\Gamma_{b}$. If the photon detector has the finer resolution then $\Gamma_{\text {expt }}=\Gamma_{\text {det }}$, the energy resolution of the detector. High purity germanium detectors seem likely to have a resolution better than or comparable to that of the light source.

The cross section for large angle elastic scattering decreases quickly with increasing photon energy. At $2 \mathrm{MeV}$, for example, the effective large angle elastic scattering cross section is about 20 times smaller than at $680 \mathrm{keV}$. In addition to the penetrability of multi-MeV gamma-rays, this is one motivation for choosing to interrogate with high energy photons. 


\subsection{Compton Backgrounds}

Depending on the count rates, it may be necessary to put a thin layer of lead or other attenuating material to filter out Compton backgrounds. Since this material would also partially filter out desired signal, system optimization could involve minimizing the needed amount of attenuation. The angle at which the photon detectors are placed (relative to the interrogation beam) can also have an important impact on system design.

Consider first the case where photon detectors are placed at $90^{\circ}$ relative to the incident beam. Photons with an energy near $680 \mathrm{keV}$ that undergo Compton scattering through 90 degrees are left with an energy of $290 \mathrm{keV}$. The total cross section (excluding coherent scattering) for $290 \mathrm{keV}$ photons in lead is about 150 barns. For $680 \mathrm{keV}$ photons the total cross section in lead is about 37 barns. This implies that the optical depth of Compton scattered photons relative to the optical depth of NRF photons in a lead attenuator is

$$
\frac{\tau_{290}^{\mathrm{Pb}}}{\tau_{680}^{\mathrm{Pb}}} \approx 4.0 .
$$

Now, the Compton scattering cross section in uranium is $\sigma_{\text {comp }} \approx 23$ barns. With the crude approximation of isotropic Compton scattering we find that the relative rate at which Compton scattered photons are observed is

$$
\frac{\lambda_{\text {Compton }}}{\lambda_{\mathrm{NRF}}}=\exp \left(-\tau_{290}^{\mathrm{Pb}}+\tau_{680}^{\mathrm{Pb}}\right) \frac{\sigma_{\text {Compton }}}{\sigma_{\mathrm{NRF}}} \times \frac{\Gamma_{b}}{\Gamma_{\mathrm{NRF}}} \approx 500 \exp \left(-3 \tau_{680}^{\mathrm{Pb}}\right) \times \frac{1 \mathrm{keV}}{\Gamma_{b}} .
$$

In writing this we have swept some fine details under the rug. The peak to total ratio for the detector and the energy dependence of the detector efficiency are both important. The numerical factor on the right hand side roughly reflects properties appropriate for a typical Ge detector.

As a second case, suppose that the photon detectors are placed at $120^{\circ}$ relative to the incident beam. Compton scattered photons entering the detector will then have an energy of $226 \mathrm{keV}$. The optical depth of lead to these photons satisfies $\tau_{226}^{\mathrm{Pb}} / \tau_{680}^{\mathrm{Pb}}=7.0$. The prefactor 3 multiplying $\tau_{680}^{\mathrm{Pb}}$ in eq. 5 then becomes a 6 if detectors are placed at $120^{\circ}$. 


\section{Design Considerations}

With an account of the collection rate for NRF and background photons in hand we can address design considerations for the T-REX source. We will only consider the case where a high resolution photon detector is used. Here a high resolution detector is one with a resolution better than or comparable to $\Gamma_{b}$.

With a high resolution notch detector one cares about elastic backgrounds and pulse pileup separately. Since elastic backgrounds cannot be distinguished from NRF photons they force a longer interrogation. Good statistics requires one to count for a time (the article submitted to JAP has a discussion of this)

$$
t_{\text {scan }} \propto \frac{1}{b}\left(1+\sqrt{\frac{\lambda_{\text {elastic }}}{\lambda_{\text {elastic }}+\lambda_{\mathrm{NRF}}}}\right)^{2}\left(1+\frac{\lambda_{\text {elastic }}}{\lambda_{\mathrm{NRF}}}\right)^{2} .
$$

When the energy resolution of the photon detector is better than that of the beam the term in parenthesis is independent of beam properties. In this case consideration of elastic backgrounds means that one should just try to maximize $b$. Even in the intermediate case where $\Gamma_{\text {det }}$ is a few times larger than $\Gamma_{\mathrm{b}}$ we could choose to count only the $635 \mathrm{keV}$ line. This choice would increase the needed experiment time by a factor of $\sim 1 / 0.56 \approx 1.75$ compared to the case where there were no elastic backgrounds. However, our best estimate for the magnitude of elastic backgrounds (eq. 3) together with eq. 6 implies that if we observe just the $680 \mathrm{keV}$ line we will already have to count for a time that is longer by a factor of about two larger than the time we would have to count were there no elastic backgrounds at all. So, from the point of view of elastic backgrounds, neither improvements in the beam resolution nor in the detector resolution will gain us much.

Whether or not Compton backgrounds are important for a high resolution detector system depends on the magnitude of the count rate $\lambda_{\text {Compton }}$. If $\lambda_{\text {Compton }} / r$ is larger than a few, i.e. if the probability of detecting a Compton photon within any given pulse is of order unity, then the Compton photons will sum with each other or with other photons and confound detection. From eqs. 5 and 1 we have that $\lambda_{\text {Compton }} / r=1$ implies that the lead attenuator must have an optical depth to $680 \mathrm{keV}$ photons equal to

$$
\tau_{680}^{\mathrm{Pb}}=\frac{1}{[4 \text { or } 7]}\left(\ln \frac{b \Gamma_{b} \epsilon_{d}}{r}-\tau_{\text {luggage }}\right) .
$$


Here the number in brackets in the denominator is 4 if photon detectors are placed at 90 degrees and 7 if photon detectors are placed at 120 degrees with respect to the incident photon beam. Of course, when this equation predicts the needed attenuator to have a negative optical depth then there is no trouble with pileup. If we are scanning luggage with an optical depth of five and if the detector system has an efficiency of $5 \%$, for example, then there is no pileup problem as long as the number of photons in each pulse of the light source is less than about 3000 .

When an attenuator is present the needed scan time scales as

$$
t_{\text {scan }} \approx \frac{1}{b \epsilon_{d}} \exp \left(\tau_{680}^{\mathrm{Pb}}+\tau_{\text {luggage }}\right)=\frac{1}{b \epsilon_{d}} \exp \left(3 \tau_{\text {luggage }} / 4\right)\left(\frac{b \Gamma_{b} \epsilon_{d}}{r}\right)^{1 / 4},
$$

if photon detectors are placed at 90 degrees. This suggests we should maximize

$$
\frac{b}{(\text { no. of photons per pulse })^{1 / 4}}
$$

If photon detectors are placed at 120 degrees, then the needed scan time scales instead at

$$
t_{\text {scan }} \propto \frac{1}{b \epsilon_{d}} \exp \left(6 \tau_{\text {luggage }} / 7\right)\left(\frac{b \Gamma_{b} \epsilon_{d}}{r}\right)^{1 / 7},
$$

which suggests we would try to maximize

$$
\frac{b}{(\text { no. of photons per pulse })^{1 / 7}} \text {. }
$$

The equations for optimizing a system that would detect a resonance at $2 \mathrm{MeV}$ (instead of the $680 \mathrm{keV}$ resonance considered here) are about the same. The energy of a $2 \mathrm{MeV}$ photon that undergoes 90 degree Compton scattering is $407 \mathrm{keV}$, while the energy of a photon scattered through 120 degrees is $291 \mathrm{keV}$. The total (excluding coherent) interaction cross section for a $2 \mathrm{MeV}$ photon in lead is about 15.6 barns, for a $291 \mathrm{keV}$ photon the interaction cross section is about 137 barns, and for a $407 \mathrm{keV}$ photon the cross section is about 71.7 barns. Taking ratios we find that the power $1 / 4$ in eq. 8 becomes $1 / 4.6$, while the power $1 / 7$ in eq. 10 becomes $1 / 8.8$ if a 2 $\mathrm{MeV}$ resonance is interrogated.

As a final remark, it is interesting to consider the virtues of a simple threshold detector. Such a detector would be insensitive to Compton 
backscattered photons and so would obviate the need for a lead attenuator. The interrogation time associated with this type of detector is simply $t_{\text {scan }} \propto\left(1 / b \epsilon_{d}\right) \exp \left(\tau_{\text {luggage }}\right)$. For $\tau_{\text {luggage }}=4$ and a system producing $10^{4}$ photons per pulse, a threshold detector could have a detection efficiency three times smaller than that of an HpGe detector while still being associated with the same scan time as the germanium detector.

\section{Summary}

If interrogation time is the principal concern, then a system seeking to detect ${ }^{238} \mathrm{U}$ via excitation of the $680 \mathrm{keV}$ state in this nucleus should be designed to have the largest possible value of

$$
\frac{b}{(\text { no. of photons per pulse) })^{\mathrm{p}}} ;\left(\begin{array}{c}
\mathrm{p}=1 / 4 \text { for detectors at } 90^{\circ} \\
\mathrm{p}=1 / 7 \text { for detectors at } 120^{\circ}
\end{array}\right) \text {. }
$$

Here $b$ is the number of photons emitted by the light source per unit energy per unit time. 